\title{
On Some Further Properties of Solutions to a Certain Semi-linear System of Partial Differential Equations
}

\author{
By \\ Atsushi Yoshikawa* and Masaya Yamaguti**
}

\section{Introduction}

We discuss the behavior of solutions to the following system of partial differential equations for $u(t, x)$ and $v(t, x)$ :

$$
\left\{\begin{array}{l}
\partial u / \partial t-\partial u / \partial x=u v, \\
\partial v / \partial t+\partial v / \partial x=-u v, \quad t>0,-\infty<x<+\infty
\end{array}\right.
$$

with the initial data:

$$
\left\{\begin{array}{l}
u(0, x)=u^{0}(x) \\
v(0, x)=v^{0}(x)
\end{array}\right.
$$

Here, $u^{0}(x)$ and $v^{0}(x)$ are supposed to be bounded, measurable, and nonnegative:

$$
0 \leqq u^{0}(x) \leqq M, 0 \leqq v^{0}(x) \leqq M, 0<M<+\infty
$$

Global existence and uniqueness of solutions to the Cauchy problem (1) (2) (3) are evident. Furthermore, $v(t, x)$ is easily seen to be bounded as $t \rightarrow+\infty$.

The system (1) can be considered as describing a development in time of two element system of prey $v(t, x)$ and predator $u(t, x)$ running on a

Communicated by S. Matsuura, March 24, 1973.

* Department of Mathematics, Hokkaido University, Sapporo. Work done while visiting the Research Institute for Mathematical Sciences, Kyoto University. Supported by the Sakkokai Foundation.

** Department of Mathematics, Kyoto University, Kyoto. 
straight line with the same speed but in the opposite directions. As to the behavior of solutions, we may state very roughly that if, at the initial stage, $u^{0}$ is spread "uniformly", then $u$ does not grow up as the time passes. On the other hand, if $u^{0}$ is "scarce", then $u$ may become arbitrarily large, though possibly spicular, as $t \rightarrow+\infty$, "wandering" to the spots where $v$ is "abundant". For the time being, we observe only partial facts which suggest the above statement.

We give some of these observations in this article. In $\S 2, \S 3$ we discuss the "uniformly" spread case. In $\S 3$, we consider the case of periodic initial data. We can then observe asymptotic periodicity in $t$ of $u(t, x)$ as $t \rightarrow+\infty$. We treat in $\S 4$ asymptotic behavior of $u$ for the case of too "abundant" $u^{0}$ with respect to $v^{0}$. In $\$ 5$ we discuss the "scarcely" spread $u^{0}$. We give several classes of $u^{0}$ and $v^{0}$, for which $u(t, x)$ does not remain bounded along a certain curve as $t \rightarrow+\infty$.

If the system (1) contained no space derivatives, then its solutions $u$ and $v$ would remain bounded under (3), for $u+v$ would then be independent of $t$. Thus our results of $\$ 5$ suggest that an interplay of the nonlinear terms and the space derivatives lets $u(t, x)$ grow exponentially under appropriate conditions. This fact is first proved by the second author (see Yamaguti [3]). As an inspection of proofs in $\$ 5$ shows, such a phenomenon essentially arises for the following system:

$$
\left\{\begin{array}{l}
\partial u / \partial t-p \partial u / \partial x=u v \\
\partial v / \partial t-q \partial v / \partial x=-u v
\end{array}\right.
$$

with $p \neq q$. Note that if $p=q$, then the system $\left(1^{\prime}\right)$ would reduce to a system of ordinary differential equations. In short, we emphasize that, though with the same non-linear terms, solutions of partial differential equations and ordinary differential equations may behave quite differently.

In passing, we note that unbounded solutions with bounded initial data also exist for general Volterra type equations:

$$
\partial u_{k} / \partial t-p_{k} \partial u_{k} / \partial x=\left(\sum_{j=1}^{N} C_{k j} u_{j}\right) u_{k}, k=1, \ldots, N .
$$

Here $p_{1}, \ldots, p_{N}$ are distinct, and

$$
C_{k j}+C_{j k}=0, \quad j, k=1, \ldots, N
$$


In fact, we may take $u_{3}=\cdots=u_{N}=0$, and then the system ( $\left.1^{\prime \prime}\right)$ is reduced to $\left(1^{\prime}\right)$.

For such considerations and background materials, we also refer to discussions on the system with confinement (Mimura [1], Mimura-KametakaYamaguti [2], Yamaguti [3]). If, for a system of semi-linear equations, there is a convex bounded set $C$ such that all the solutions starting from $C$ always lie in $C$, then the system is said to be with confinement. If non-linear terms are of quadratic forms, a system with confinement is characterized by means of its coefficients $([1],[3])$. Thus the system (1") is with confinement only when all the $p_{k}{ }^{\prime}$ s are identical. If the $p_{k}{ }^{\prime} \mathrm{s}$ are distinct we cannot even find two bounded sets $B$ and $B^{\prime}$ such that any solution starting from $B$ remains in $B^{\prime}$.

\section{The Uniformly Spread Case}

In order to avoid the trivial case, we assume throughout the paper that $u^{0}$ and $v^{0}$ do not vanish identically.

We will often denote $\frac{1}{2} \int_{b}^{a} u^{0}(r) d r$ by $\phi(a, b)$ :

$$
\phi(a, b)=\frac{1}{2} \int_{b}^{a} u^{0}(r) d r
$$

Definition 2.1. We say that $u^{0}(x)$ is uniformly spread if the set $\{\exp \{-\phi(a, a-s)\}:-\infty<a<+\infty\}$ is uniformly bounded in $L^{1}(0,+\infty)$.

Proposition 2.2. If $u^{0}(x)$ is uniformly spread, then for every $x^{0}$, $-\infty<x^{0}<+\infty, u^{0}(x) \notin L^{1}\left(-\infty, x^{0}\right)$ and $u^{0}(x) \notin L^{1}\left(x^{0},+\infty\right)$.

Proof. If $u^{0}(x) \in L^{1}\left(-\infty, x^{0}\right)$, then

$$
\begin{aligned}
\phi(a, a-s) & =\frac{1}{2} \int_{a-s}^{a} u^{0}(r) d r \\
& \leqq \frac{1}{2} \int_{-\infty}^{a} u^{0}(r) d r=C(a) .
\end{aligned}
$$

Thus, as functions in $s$,

$$
\exp \{-\phi(a, a-s)\} \geqq e^{-C(a)} \notin L^{1}(0,+\infty) .
$$


If $u^{0}(x) \in L^{1}\left(x^{0},+\infty\right)$, then by setting $\psi(a)=\frac{1}{2} \int_{a}^{\infty} u^{0}(x) d x$, we should have $\phi(a, b)=\psi(b)-\psi(a)$. Since $u^{0}(x)$ is uniformly spread, this would imply that

$$
\int_{-\infty}^{a} e^{-\psi(s)} d s \leqq C e^{-\psi(a)}
$$

for all $a$ with some constant $C>0$. This is, however, impossible. In fact, then for any $a>0$

$$
\begin{aligned}
e^{-\psi(0)} a & \leqq \int_{0}^{a} e^{-\psi(s)} d s \leqq C e^{-\psi(a)} \\
& \leqq C
\end{aligned}
$$

since $\psi(s) \leqq \psi(0)$ if $s \geqq 0$.

Q.E.D.

Remark 2.3. If there is a non-negative function $\phi_{1}(s)$ with

$$
\frac{1}{2} \int_{s}^{a} u^{0}(r) d r \geqq \phi_{1}(a-s), \quad a>s,
$$

and $\exp \left(-\phi_{1}(s)\right) \in L^{1}(0,+\infty)$, then $u^{0}(x)$ is uniformly spread.

By this remark, we can prove

Proposition 2.4. If $u^{0}(x)$ is periodic and $u^{0}(x) \not \equiv 0$, then $u^{0}(x)$ is uniformly spread. More precisely, as $\phi_{1}(s)$ in Remark 2.3, we can take

$$
\phi_{1}(s)= \begin{cases}0, & s<\sigma, \\ m s / 2 \sigma-m / 2, & s \geqq \sigma .\end{cases}
$$

Here $\sigma$ is the period of $u^{0}(x)$ and $m=\int_{0}^{\infty} u^{0}(r) d r$.

Proof. By the periodicity of $u^{0}$, we have for any $s>0$ and real $b$

$$
\frac{1}{2} \int_{b}^{b+s} u^{0}(r) d r \geqq \frac{1}{2} k \int_{b}^{b+\infty} u^{0}(r) d r=\frac{1}{2} k m
$$

if $k$ is the integral part of $s / \tau$. Since $k>s / \sigma-1$, we have 


$$
\frac{1}{2} \int_{b}^{b+s} u^{0}(r) d r \geqq m s / 2 \sigma-m / 2, s \geqq \sigma .
$$

Remark 2.5. If $u^{0}(x) \geqq a>0$, then $u^{0}(x)$ is uniformly spread with $\phi_{1}(s)=a s, s \geqq 0$.

Proposition 2.6. If $u^{0}(x)$ is uniformly spread, then the solutions to the Cauchy problem (1) (2) (3) remain bounded as $t \rightarrow+\infty$.

Proof. The system (1) (2) is immediately seen to be equivalent to the following system:

(4)

$$
\left\{\begin{array}{l}
u(t, x)= \\
\quad u^{0}(x+t) \exp \left\{\int_{0}^{t} v^{0}(x+t-2 \tau) \exp \left[-\int_{0}^{\tau} u(\sigma, x+t-2 \tau+\sigma) d \sigma\right] d \tau\right\}, \\
v(t, x)= \\
v^{0}(x-t) \exp \left\{-\int_{0}^{t} u^{0}(x-t+2 \sigma) \exp \left[\int_{0}^{\sigma} v(\tau, x-t+2 \sigma-\tau) d \tau\right] d \sigma\right\} .
\end{array}\right.
$$

Under the condition (3), we immediately have

$$
0 \leqq v(t, x) \leqq v^{0}(x-t) \leqq M .
$$

It remains to show the boundedness of $u(t, x)$. From (4),

$$
u(t, x) \geqq u^{0}(x+t) .
$$

Substituting (5) into (4), we have

$$
\begin{gathered}
u(t, x) \leqq u^{0}(\xi) \exp V(\xi, \eta), \quad \xi=x+t, \eta=x-t, \\
V(\xi, \eta)=\frac{1}{2} \int_{\eta}^{\xi} v^{0}(\theta) \exp \left(-\frac{1}{2} \int_{\eta}^{\xi} u^{0}(\sigma) d \sigma\right) d \theta .
\end{gathered}
$$

Since $v^{0}(\theta) \leqq M$ by the assumptions and since $u^{0}$ is uniformly spread,

$$
\begin{aligned}
V(\xi, \eta) & \leqq \frac{1}{2} M \int_{\eta}^{\xi} \exp (-\phi(\xi, \theta)) d \theta \\
& =\frac{1}{2} M \int_{0}^{2 t} \exp (-\phi(\xi, \xi-\theta)) d \theta \leqq \frac{1}{2} M C .
\end{aligned}
$$


Hence,

$$
u(t, x) \leqq M \exp \left(\frac{1}{2} M C\right)<+\infty
$$

for all $t>0,-\infty<x<+\infty$.

Q.E.D.

Remark 2.7. The systems (1)-(2) or (4) are also equivalent to the following:

$$
\left\{\begin{array}{l}
u(t, x)=u^{0}(x+t) \exp \left(\int_{0}^{t} v(s, x+t-s) d s\right), \\
v(t, x)=v^{0}(x-t) \exp \left(-\int_{0}^{t} u(s, x-t+s) d s\right) .
\end{array}\right.
$$

Remark 2.8. In the proof of proposition 2.6, we have essentially shown that $\{v(x, a-s) ;-\infty<a<+\infty\}$ is a bounded set in $L^{1}(0,+\infty)$ if $u^{0}$ is uniformly spread. In fact, by (4) we have

$$
\begin{aligned}
& v(t, x) \leqq v^{0}(\eta) \exp \left(-\frac{1}{2} \int_{\eta}^{\xi} u^{0}(\theta) d \theta\right), \\
& \xi=x+t, \eta=x-t .
\end{aligned}
$$

\section{The Case of Periodic Initial Data}

In this section we assume that $u^{0}$ and $v^{0}$ are both non-trivial nonnegative periodic functions of the same period $\sigma$.

Since the initial value problem (1) (2) (3) is uniquely solved and since the system (1) is invariant by translation, we have

Proposition 3.1. If $u^{0}(x)$ and $v^{0}(x)$ are periodic of period $\sigma$, then the solutions $u(t, x)$ and $v(t, x)$ of the initial value problem (1) (2) (3) are periodic in $x$ of period $\sigma$ :

$$
u(t, x+\varpi)=u(t, x), v(t, x+\sigma)=v(t, x) .
$$

Moreover, $u(t, x)$ is asymptotically periodic in $t$ as seen from

Proposition 3.2. Under the assumptions of Proposition 3.1, 


$$
u(t, x) \leqq u(t+\sigma, x) \leqq e^{\varpi(t)} u(t, x) \quad \text { for } \quad t \geqq \sigma / 2 .
$$

Here

$$
\Phi(t)=n \exp (m / 2-m t / \sigma) \rightarrow 0 \quad \text { as } \quad t \rightarrow+\infty
$$

and

$$
m=\int_{0}^{\pi} u^{0}(r) d r, n=\int_{0}^{\sigma} v^{0}(r) d r
$$

Proof. From (4) we have, using Proposition 3.1,

$$
\begin{aligned}
& u(t+\sigma, x)=u(t, x) \exp \Phi(x, t) \\
& \Phi(x, t)=\int_{t}^{t+\sigma} v^{0}(x+t-2 \tau) \exp \left[-\int_{0}^{\tau} u(\sigma, x+t-2 \tau+\sigma) d \sigma\right] d \tau .
\end{aligned}
$$

From (5),

$$
\begin{aligned}
& \Phi(x, t) \leqq \frac{1}{2} \int_{\eta-2{ }^{\sigma}}^{\eta} v^{0}(\rho) \exp \left[-\frac{1}{2} \int_{\rho}^{\xi} u^{0}(\sigma) d \sigma\right] d \rho, \\
& \eta=x-t, \xi=x+t=\eta+2 t
\end{aligned}
$$

If $2 t \geqq \varpi$, then by Proposition 2.4 ,

$$
\begin{gathered}
\Phi(x, t) \leqq \frac{1}{2} e^{m / 2-m t / \sigma} \int_{\eta-2^{\sigma}}^{\eta} v^{0}(\rho) e^{-m(\eta-\rho) / 2^{\sigma}} d \rho \\
\leqq n \exp (m / 2-m t / \sigma)=\Phi(t) .
\end{gathered}
$$

Since $\Phi(x, t) \geqq 0$, we have proved the proposition.

Q.E.D.

On the contrary, $v(t, x)$ enjoys the following decay property. Namely, we have

Proposition 3.3. Under the assumptions of Proposition 3.1, we have

$$
e^{-K} v(t, x) \leqq v(t+\sigma, x) \leqq e^{-m} v(t, x), t \geqq \sigma / 2 \text {. }
$$

Here $K=m \exp \left\{n\left(2-e^{-m / 2}\right) / 2\left(1-e^{-m / 2}\right)\right\}, m, n$ being as in Proposition 3.2 . 
Proof. From (4) we have, using Proposition 3.1,

$$
\begin{aligned}
v(t, x) & =v(t+\sigma, x) \exp \Psi(t, x) \\
\Psi(t, x) & =\int_{t}^{t+\sigma} u^{0}(x-t+2 \sigma) \exp \left[\int_{0}^{\sigma} v(\tau, x-t+2 \sigma-\tau) d \tau\right] d \sigma .
\end{aligned}
$$

It suffices to show the following inequality:

(8)

$$
m \leqq \Psi(t, x) \leqq K
$$

Since $v(t, x) \geqq 0$, we have

$$
\Psi(t, x) \geqq \int_{t}^{t+\infty} u^{0}(x-t+2 \sigma) d \sigma=m
$$

Now we are going to show the right-hand side of (8). From (4)

$$
\begin{aligned}
& v(t, x) \leqq v^{0}(\eta) \exp \left(-\frac{1}{2} \int_{\eta}^{\xi} u^{0}(\sigma) d \sigma\right), \\
& \eta=x-t, \xi=x+t
\end{aligned}
$$

Since

$$
\frac{1}{2} \int_{\eta}^{\xi} u^{0}(\sigma) d \sigma \geqq m t / \sigma-m / 2,2 t \geqq \sigma,
$$

we have

$$
v(t, x) \leqq\left\{\begin{array}{l}
v^{0}(\eta), t<\sigma / 2, \\
v^{0}(\eta) \exp (-m t / \sigma+m / 2), t \geqq \sigma / 2 .
\end{array}\right.
$$

Thus, for $\sigma \geqq t \geqq \sigma / 2$,

$$
\int_{0}^{\sigma} v(\tau, \eta+2 \sigma-\tau) d \tau \leqq I_{1}+I_{2},
$$

where

$$
I_{1}=\int_{0}^{\pi / 2} v^{0}(\eta+2 \sigma-2 \tau) d \tau
$$

and

$$
I_{2}=\int_{\varpi / 2}^{\sigma} v^{0}(\eta+2 \sigma-2 \tau) e^{-m \tau / \sigma+m / 2} d \tau
$$


By the periodicity of $v^{0}, I_{1}=n / 2$. On the other hand,

$$
\begin{aligned}
I_{2} & =\int_{0}^{\sigma-\sigma / 2} v^{0}(\eta+2 \sigma-2 \tau) e^{-m \tau / \sigma} d \tau \\
& \leqq \frac{1}{2} \int_{0}^{\infty} v^{0}\left(\eta_{1}-\theta\right) e^{-m \theta / 2 \sigma} d \theta, \eta_{1}=\eta+2 \sigma, \\
& \leqq \frac{1}{2} \sum_{k=0}^{\infty} e^{-m k / 2} \int_{0}^{\sigma} v^{0}\left(\eta_{1}-\theta\right) e^{-m \theta / 2 \sigma} d \theta \\
& \leqq \frac{1}{2} n\left(1-e^{-m / 2}\right)^{-1}
\end{aligned}
$$

Thus

$$
\int_{0}^{\sigma} v(\tau, \eta+2 \sigma-\tau) d \tau \leqq \frac{1}{2} n\left(2-e^{-m / 2}\right)\left(1-e^{-m / 2}\right)^{-1}=K^{\prime}
$$

Hence,

$$
\Psi(t, x) \leqq e^{K^{\prime}} \int_{t}^{t+\infty} u^{0}(\eta+2 \sigma) d \sigma=m e^{K^{\prime}}=K
$$

and the inequality (8) is proved.

Q.E.D.

\section{A Starvation Phenomenon}

By inspecting the first equation of (4), we immediately have

Proposition 4.1. If $v^{0}(x) \in L^{1}(-\infty,+\infty)$, then the solutions $u(t, x)$ and $v(t, x)$ of the initial value problem (1) (2) (3) remain bounded as $t \rightarrow+\infty$.

Proof. It suffices to show the boundedness of $u(t, x)$. From (4),

$$
\begin{aligned}
& u(t, x) \leqq u^{0}(\xi) \exp \left(\frac{1}{2} \int_{\eta}^{\xi} v^{0}(\theta) d \theta\right), \\
& \xi=x+t, \eta=x-t .
\end{aligned}
$$

Hence

$$
u(t, x) \leqq M \exp \left(\frac{1}{2} \int_{-\infty}^{\infty} v^{0}(\theta) d \theta\right)<+\infty
$$


The following propositions suggest a "starvation" of $u$ in case when $u^{0}$ is abundant while $v^{0}$ is running short.

Proposition 4.2. Let $v^{0}(x) \in L^{1}(-\infty,+\infty)$ such that $v^{0}(x)=0, x>a$, for some $a\left(v^{0}(x) \not \equiv\right)$. If $u^{0}(x) \notin L^{1}(a,+\infty)$, then for every $c,-1<c \leqq 1$, and $x^{0},-\infty<x^{0}<+\infty$, we have

$$
u^{0}(\xi(t)) \leqq u\left(t, c t+x^{0}\right) \leqq e^{\alpha(t)} u^{0}(\xi(t))
$$

for large $t$. Here $\xi(t)=(1+c) t+x^{0}, \alpha(t)=\alpha\left(t ; c, x^{0}, u^{0}, v^{0}\right) \geqq 0$, and $\alpha(t) \rightarrow 0$ as $t \rightarrow+\infty$.

Proof. Since

$$
\frac{1}{2} \int_{\theta}^{\xi} u^{0}(\sigma) d \sigma \geqq \frac{1}{2} \int_{a}^{\xi} u^{0}(\sigma) d \sigma=\phi(\xi, a)
$$

for $\xi \geqq a \geqq \theta$, we have by (7) and our assumptions on $v^{0}$ that

$$
V(\xi, \xi-2 t) \leqq \frac{1}{2} \int_{\xi-2 t}^{a} v^{0}(\theta) d \theta \exp (-\phi(\xi, a))
$$

if $\xi-2 t \leqq a$. Hence, by (6)

$$
u(t, x) \leqq u^{0}(\xi) \exp \left\{\frac{1}{2} \int_{\xi-2 t}^{a} v^{0}(\theta) d \theta \exp (-\phi(\xi, a))\right\}
$$

for $\xi=x+t \geqq a$ and $\xi-2 t \leqq a$. If $x=c t+x^{0},-1<c<1$, then $\xi(t)=$ $(1+c) t+x^{0} \rightarrow+\infty$, and $\xi(t)-2 t \rightarrow-\infty$ as $t \rightarrow+\infty$. On the other hand, since $u^{0}(\sigma) \notin L^{1}(a,+\infty), \phi(\xi(t), a) \rightarrow+\infty, t \rightarrow+\infty$. Hence,

$$
\begin{aligned}
& \alpha(t)=\frac{1}{2} \int_{-\infty}^{a} v^{0}(\theta) d \theta \exp (-\phi(\xi(t), a)) \rightarrow 0, \\
& t \rightarrow+\infty
\end{aligned}
$$

and

$$
u\left(t, c t+x^{0}\right) \leqq u^{0}(\xi(t)) e^{\alpha(t)} .
$$

Combining this with (5), we have (10). If $c=1$, then $V(\xi(t), \xi(t)-2 t)=0$ for $x^{0} \geqq a$, and $V(\xi(t), \xi(t)-2 t) \leqq \frac{1}{2} \int_{x^{0}}^{a} v^{0}(\theta) d \theta \exp (-\phi(\xi(t), a))$ for $x^{0}<a$. 
Hence, (10) holds also good for this case.

Q.E.D.

Proposition 4.3. Take a non-negative differentiable function $k(x)$ in $x \geqq a$ such that $\lim _{x \rightarrow+\infty} x e^{-k(x)}=0 . \quad$ Let $v^{0}(x) \in L^{1}(-\infty,+\infty)$ with $v^{0}(x) \leqq$ $M e^{-k(x)}$ for $x \geqq a$. If $m(x)=\max \left(0,2 k^{\prime}(x)-u^{0}(x)\right) \in L^{1}(a,+\infty)$, then the same conclusion as in Proposition 4.2. holds with $c,-1<c<1$.

Proof. First, we note $u^{0}(x) \notin L^{1}(a,+\infty)$. For if $x>a$,

$$
\int_{a}^{x}\left(2 k^{\prime}(r)-u^{0}(r)\right) d r \leqq \int_{a}^{\infty} m(r) d r<+\infty,
$$

whence, as $x \rightarrow+\infty$,

$$
\int_{a}^{x} u^{0}(r) d r \geqq 2 k(x)-2 k(a)-\int_{a}^{\infty} m(r) d r \rightarrow+\infty .
$$

By the proof of Proposition 4.2, it suffices to show

$$
V(\xi(t), \xi(t)-2 t) \rightarrow 0 \quad \text { as } \quad t \rightarrow+\infty .
$$

In fact, for large $t$, we have

$$
\begin{aligned}
& V(\xi(t), \xi(t)-2 t) \\
& \leqq \\
& \quad \frac{1}{2} \int_{\xi(t)-2 t}^{a} v^{0}(\theta) d \theta \exp \left(-\frac{1}{2} \int_{a}^{\xi(t)} u^{0}(\sigma) d \sigma\right) \\
& \quad+\frac{1}{2} M \int_{a}^{\xi(t)} \exp \left\{-k(\theta)-\frac{1}{2} \int_{\theta}^{\xi(t)} u^{0}(\sigma) d \sigma\right\} d \theta .
\end{aligned}
$$

Since

$$
\begin{aligned}
-k(\theta) & -\frac{1}{2} \int_{\theta}^{\xi(t)} u^{0}(\sigma) d \sigma \\
& =-k(\xi(t))+\int_{\theta}^{\xi(t)}\left(k^{\prime}(\sigma)-\frac{1}{2} u^{0}(\sigma)\right) d \sigma \\
& \leqq-k(\xi(t))+\frac{1}{2} \int_{a}^{\infty} m(\sigma) d \sigma
\end{aligned}
$$

we have 


$$
\begin{aligned}
& \frac{1}{2} M \int_{a}^{\xi(t)} \exp \left\{-k(\theta)-\frac{1}{2} \int_{\theta}^{\xi(t)} u^{0}(\sigma) d \sigma\right\} d \theta \\
& \quad \leqq \frac{1}{2} M\left\{\exp \left(\frac{1}{2} \int_{a}^{\infty} m(\sigma) d \sigma\right)\right\}(\xi(t)-a) e^{-k(\xi(t))} .
\end{aligned}
$$

Consequently,

$$
V(\xi(t), \xi(t)-2 t) \rightarrow 0 \quad \text { as } \quad t \rightarrow+\infty .
$$

Q.E.D.

\section{The Case of Not Uniformly Spread $u^{0}$}

If $u^{0}(x)=0$ for $x<x^{0}$, then $u^{0}(x)$ cannot be uniformly spread by Proposition 2.2. Noting this and Proposition 4.1, we see the following proposition is of interest.

Proposition 5.1. Assume that $u^{0}(x)=0, x<x^{0}$, and that $\left(x-x^{0}\right)^{-\gamma}$ $u^{0}(x)$ tends to $c^{0}>0$ as $x \downarrow x^{0}$ for some $\gamma, 0 \leqq \gamma<+\infty$. If $v^{0}(x) \notin L^{1}(-\infty$, $\left.x^{0}\right)$, then $u(t, x)$ does not remain bounded as $t \rightarrow+\infty$. More precisely, there is a function $\psi(\xi)$ such that $\psi(\xi) \rightarrow+\infty$ as $\xi \downarrow x^{0}$,

$$
\begin{aligned}
& \frac{1}{2} \int_{-\psi(\xi)}^{x^{0}} v^{0}(\sigma) d \sigma \geqq(\gamma+1) \log \left(\xi-x^{0}\right)^{-1}-\log \frac{1}{4} c^{0}, \\
& \xi \downarrow x^{0},
\end{aligned}
$$

and that

$$
\begin{aligned}
& u(t, x) \rightarrow+\infty, t=(\xi+\psi(\xi)) / 2, \\
& x=(\xi-\psi(\xi)) / 2, \xi \downarrow x^{0} .
\end{aligned}
$$

Proof. We may assume $x^{0}=0$. Let $\xi=x+t, \eta=x-t$ as before. Substituting (9) into (4), we have

$$
u(t, x) \geqq u^{0}(\xi) \exp \left\{\frac{1}{2} \int_{\eta}^{\xi} v^{0}(\theta) e^{-f(\xi, \theta)} d \theta\right\},
$$

where

$$
f(\xi, \theta)=\frac{1}{2} \int_{\theta}^{\xi} u^{0}(\rho) \exp \left(\frac{1}{2} \int_{\theta}^{\rho} v^{0}(\sigma) d \sigma\right) d \rho
$$


By the assumptions on the support of $u^{0}$, it suffices to discuss the behavior of $u(t, x)$ for $\xi \geqq 0$. For $\xi \geqq 0, \theta<0$, we have

$$
f(\xi, \theta)=\frac{1}{2} \int_{0}^{\xi} u^{1}(\rho) \exp \left(\frac{1}{2} \int_{\theta}^{0} v^{0}(\sigma) d \sigma\right) d \rho,
$$

where

$$
u^{1}(\rho)=u^{0}(\rho) \exp \left(\frac{1}{2} \int_{0}^{\rho} v^{0}(\sigma) d \sigma\right)
$$

Therefore, by setting

$$
\alpha(\xi)=\frac{1}{2} \int_{0}^{\xi} u^{1}(\rho) d \rho, \xi \geqq 0,
$$

and

$$
\beta(\theta)=\exp \left(\frac{1}{2} \int_{\theta}^{0} v^{0}(\sigma) d \sigma\right), \theta<0
$$

we have

$$
f(\xi, \theta)=\alpha(\xi) \beta(\theta), \xi \geqq 0, \theta<0 .
$$

Thus if $\eta<0$,

$$
\begin{aligned}
\frac{1}{2} \int_{\eta}^{\xi} v^{0}(\theta) e^{-f(\xi, \theta)} d \theta & \geqq \frac{1}{2} \int_{\eta}^{0} v^{0}(\theta) e^{-a(\xi) \beta(\theta)} d \theta \\
& =\int_{1}^{\beta(\eta)} e^{-\alpha(\xi) r} d r / r .
\end{aligned}
$$

Here we have used the transformation $r=\beta(\theta)$ and $d r / r=-\frac{1}{2} v^{0}(\theta) d \theta$. Since $v^{0} \notin L^{1}(-\infty, 0)$, there exists a function $\psi(\xi)$ satisfying (11) (with $\left.x^{0}=0\right)$. In reality, it is enough for our purpose that (11) holds for a sequence $\xi_{n} \downarrow 0$. Now by the definition of $\alpha(\xi), \xi^{-\gamma-1} \alpha(\xi) \rightarrow \frac{1}{2} c^{0}$ as $\xi \downarrow 0$ since $x^{-\gamma} u^{0}(x) \rightarrow c^{0}$ as $x \downarrow 0$. Thus, from (11), we have

$$
\alpha(\xi) \beta(-\psi(\xi)) \geqq 1, \xi \downarrow 0 .
$$

Now choose $\varepsilon, 0<\varepsilon \leqq 1$, so that

$$
0 \leqq \gamma<\left(e^{\varepsilon}-1\right)^{-1}
$$


Since $\alpha(\xi) \downarrow 0$ as $\xi \downarrow 0$, we have by (14) and (15)

$$
\begin{aligned}
& \frac{1}{2} \int_{-\psi(\xi)}^{\xi} v^{0}(\theta) e^{-f(\xi, \theta)} d \theta \\
& \geqq \int_{1}^{\beta(-\psi(\xi))} e^{-\alpha(\xi) r} d r / r \\
& \quad=\int_{\alpha(\xi)}^{\alpha(\xi) \beta(-\psi(\xi))} e^{-r} d r / r \\
& \geqq \int_{\alpha(\xi)}^{\varepsilon} e^{-r} d r / r \geqq e^{-\varepsilon} \int_{\alpha(\xi)}^{\varepsilon} d r / r .
\end{aligned}
$$

Thus,

$$
\begin{aligned}
& \frac{1}{2} \int_{-\psi(\xi)}^{\xi} v^{0}(\theta) e^{-f(\xi, \theta)} d \theta \\
& \quad \geqq e^{-\varepsilon}\left(\log \alpha(\xi)^{-1}-\log \varepsilon^{-1}\right), \xi \downarrow 0 .
\end{aligned}
$$

Hence, for $t=(\xi+\psi(\xi)) / 2, x=(\xi-\psi(\xi)) / 2$, we have by (12)

$$
\begin{aligned}
u(t, x) & \geqq u^{0}(\xi) \exp \left\{\frac{1}{2} \int_{-\psi(\xi)}^{\xi} v^{0}(\theta) e^{-f(\xi, \theta)} d \theta\right\} \\
& \geqq u^{0}(\xi) \exp \left\{e^{-\varepsilon}\left[\log \alpha(\xi)^{-1}-\log \varepsilon^{-1}\right]\right. \\
& =C_{\varepsilon} u^{0}(\xi) \alpha(\xi)^{e^{-\varepsilon}}, \\
C_{\varepsilon} & =\varepsilon^{e^{-\varepsilon}}>0, \xi \downarrow 0 .
\end{aligned}
$$

However, for some $c^{1}>0$,

$$
u^{0}(\xi) \alpha(\xi)^{-e^{-\ell}} \xi^{\delta} \rightarrow c^{1} \quad \text { as } \quad \xi \downarrow 0
$$

with $\delta=-\gamma+e^{-\varepsilon}(\gamma+1)=e^{-\varepsilon}\left\{1-\left(e^{\varepsilon}-1\right) \gamma\right\}>0$ by the choice of $\varepsilon$. Consequently, as $\xi \downarrow 0$,

$$
u(t, x) \rightarrow+\infty, t=(\xi+\psi(\xi)) / 2, x=(\xi-\psi(\xi)) / 2 .
$$

Q.E.D.

Remark 5.2. Proposition 5.1 was inspired by the case when $u^{0}(x)$ $=0, x<0, u^{0}(x)=1, x \geqq 0 ; v^{0}(x)=1, x<0, v^{0}(x)=0, x \geqq 0$, given originally 
by Kametaka. Generally speaking, $u^{0}(x)$ as satisfying the assumptions of Proposition 5.1 cannot be infinitely differentiable at $x^{0}$. We do not know whether the proposition holds good when $u^{0}(x)$ is infinitely differentiable (in particular, when $x^{0}$ is a zero of the infinite order of $u^{0}(x)$ ).

In order to treat another class of not uniformly spread $u^{0}$, we introduce the following function $\tilde{\phi}_{1}(r),-\infty<r<+\infty$, by

$$
\tilde{\phi}_{1}(r)=\frac{1}{2} \int_{-\infty}^{r} u^{0}(s) e^{V s} d s
$$

for some fixed positive constant $V$. If $v^{0}(x) \leqq 2 V$, then we have by (13)

$$
f(\xi, \theta) \leqq e^{-V \theta} \tilde{\phi}_{1}(\xi) \equiv \tilde{\phi}(\xi, \theta)
$$

for $\theta<\xi$, where the last equality for the definition.

Remark 5.3. The function $\tilde{\phi}(\xi, \theta)$ gives a good information for the estimate of $u(t, x)$ from below. In fact, if $0 \leqq v^{0}(x) \leqq 2 V$, we have by (12) and (13)

$$
u(t, x) \geqq u^{0}(\xi) \exp \left\{\frac{1}{2} \int_{\eta}^{\xi} v^{0}(\theta) e^{-f^{\prime}(\xi, \theta)} d \theta\right\}
$$

where

$$
\begin{aligned}
& f^{\prime}(\xi, \theta)=\frac{1}{2} \int_{\theta}^{\xi} u^{0}(\rho) e^{(\rho-\theta) V} d \rho, \\
& \xi=x+t, \eta=x-t .
\end{aligned}
$$

If $0 \leqq u^{0}(x) \leqq M$, we have

$$
\tilde{\phi}(\xi, \theta)-\frac{1}{2} M / V \leqq f^{\prime}(\xi, \theta) \leqq \tilde{\phi}(\xi, \theta)
$$

since

$$
0 \leqq e^{-\theta V} \tilde{\phi}_{1}(\theta) \leqq \frac{1}{2} M / V
$$

In particular, we have 


$$
\begin{aligned}
& u(t, x) \geqq u^{0}(\xi) \exp F(\xi, \eta), \\
& F(\xi, \eta)=\frac{1}{2} \int_{\eta}^{\xi} v^{0}(\theta) e^{-\tilde{\phi}(\xi, \theta)} d \theta .
\end{aligned}
$$

Now since

$$
\phi(\xi, \theta)=\frac{1}{2} \int_{\theta}^{\xi} u^{0}(\rho) d \rho \leqq f^{\prime}(\xi, \theta) \leqq \tilde{\phi}(\xi, \theta),
$$

we see that if

$$
\lim _{\xi \rightarrow+\infty} \inf \tilde{\phi}_{1}(\xi) e^{-V \xi}=0
$$

then $u^{0}$ cannot be uniformly spread. In fact, then

$$
\begin{aligned}
& \int_{0}^{\infty} \exp \{-\phi(\xi, \xi-\theta)\} d \theta \\
& \left.\geqq \int_{0}^{\infty} \exp \{-\tilde{\phi}(\xi, \xi-\theta)\} d \theta\right) \\
& \quad=\int_{C(\xi)}^{\infty} e^{-r} d r / r \\
& C(\xi)=\tilde{\phi}_{1}(\xi) e^{-V \xi},
\end{aligned}
$$

and the last integral tends to $+\infty$ as $\xi \rightarrow+\infty$. Consequentely, if in particular $u^{0}(x) e^{V x} \in L^{1}(-\infty,+\infty)$, then $u^{0}$ is not uniformly spread (cf. also Proposition 2.2). Then we have the following

Proposition 5.4. Suppose that there be a function $\xi(t)$ such that $\xi(t) \rightarrow+\infty, \xi(t)-2 t \rightarrow-\infty, t \rightarrow+\infty, \liminf _{t \rightarrow+\infty} \tilde{\phi}_{1}(\xi(t)) e^{-\xi(t) V}=0$, and that $\limsup _{t \rightarrow+\infty} u^{0}(\xi(t)) e^{\xi(t) V} / \tilde{\phi}_{1}(\xi(t))=+\infty$. Then for any $v^{0}(x), 0 \leqq v^{0}(x) \leqq 2 V$, with $v^{0}(x) \geqq \alpha>0, x>x^{0}$, we have

$$
\lim _{t \rightarrow+\infty} \sup u(t, \xi(t)-t)=+\infty
$$

Proof. Since $0 \leqq v^{0}(x) \leqq 2 V$, we can apply (18). Putting $\rho(\theta)=e^{-V \theta}$, we have

$$
F(\xi, \eta)=(2 V)^{-1} \int_{\rho(\xi)}^{\rho(\eta)} \tilde{v}^{0}(\rho) \exp \left\{-\rho \phi_{1}(\xi)\right\} d \rho / \rho
$$


where

$$
\tilde{v}^{0}(\rho)=v^{0}\left(V^{-1} \log \rho^{-1}\right)
$$

By (19), if $\xi-2 t<x$, and $\xi \geqq 0$,

$$
F(\xi, \xi-2 t) \geqq(2 V)^{-1} \alpha \int_{c(\xi)}^{\beta} e^{-r} d r / r
$$

$c(\xi)=e^{-V \xi} \tilde{\phi}_{1}(\xi), \beta=e^{-V x^{0}} \tilde{\phi}_{1}(0)$. Hence, by our assumptions,

$$
F(\xi(t), \xi(t)-2 t) \geqq C_{1} \log c(\xi(t))^{-1}+c_{2}
$$

for large $t, C_{1}=\alpha e^{-\beta} / 2 V, C_{2}=C_{1} \log \beta$.

Consequently, by (18) we have

$$
u(t, \xi(t)-t) \geqq C_{3} u^{0}(\xi(t)) e^{\xi(t) V} / \tilde{\phi}_{1}(\xi(t)), C_{3}>0 .
$$

Q.E.D.

Specializing the assumptions of the previous proposition, we have the following more precise version of Yamaguti's result ([3]).

Proposition 5.5. Suppose $u^{0}(x) e^{V x} \in L^{1}(-\infty,+\infty)$ and $v^{0}(x) \leqq 2 V$, $V>0$. Assume that there be a sequence $\xi_{n} \rightarrow+\infty$ such that

$$
u^{0}\left(\xi_{n}\right) \exp \left(C_{1} \int_{\eta^{0}}^{\xi_{n}} v^{0}(\theta) d \theta\right) \rightarrow+\infty
$$

for some real $\eta^{0}$ and positive $C_{1} \leqq(2 V)^{-1} \exp \left(-e^{-V \eta^{0}} \int_{-\infty}^{\infty} u^{0}(x) e^{V x} d x\right)$. Then $u\left(t_{n}, x_{n}\right) \rightarrow+\infty, n \rightarrow+\infty, t_{n}=\left(\xi_{n}-\eta\right) / 2, x_{n}=\left(\xi_{n}+\eta\right) / 2$ for every fixed $\eta \leqq \eta^{0}$. In particular, if there is a sequence $\xi_{n} \rightarrow+\infty$ such that $u^{0}\left(\xi_{n}\right) \geqq \delta>0$, then for any $v^{0} \notin L^{1}\left(\eta^{0},+\infty\right), v^{0}(x) \leqq 2 V, u\left(t_{n}, x_{n}\right) \rightarrow+\infty, t_{n}=\left(\xi_{n}-\eta\right) / 2$, $x_{n}=t_{n}+\eta$, with a fixed $\eta \leqq \eta^{0}$.

Proof. Since $u^{0}(x) e^{V x} \in L^{1}(-\infty, \infty), 0 \leqq v^{0}(x) \leqq 2 V$, and $\eta \leqq \eta^{0}$, we have by (19)

$$
\begin{aligned}
F(\xi, \eta) & \geqq C_{1} \int_{\rho(\xi)}^{\rho\left(\eta^{0}\right)} \widetilde{v}^{0}(\rho) d \rho / \rho \\
& =C_{1} \int_{\eta^{0}}^{\xi} v^{0}(\theta) d \theta
\end{aligned}
$$


Here $C_{1}=e^{-c / 2 V}, c \geqq \rho\left(\eta^{0}\right) \sup _{\gamma} \tilde{\phi}_{1}(\gamma)$. Substituting (20) into (18), we have

$$
u(t, x) \geqq u^{0}(\xi) \exp \left(C_{1} \int_{\eta^{0}}^{\xi} v^{0}(\theta) d \theta\right)
$$

$\xi=x+t, \eta=x-t \leqq \eta^{0}$. This inequality immediately implies the proposition.

Q.E.D.

Finally we give examples, as illustrations to Propositions 5.4 and 5.5.

Example 5.6. Take a function $a(x)$ supported in $[-1,1]$ such that $a(0)=1$ and $0 \leqq a(x) \leqq 1$. Put

$$
u^{0}(x)= \begin{cases}0, & x<1 \\ a\left((x-2 n) / d_{n}\right), & 2 n-1 \leqq x \leqq 2 n+1,\end{cases}
$$

with $d_{n} \leqq 1$. Thus $u^{0}(2 n)=1$. If $d_{n}=e^{-\delta n}, n=1,2, \ldots$, with any positive $\delta$, then $u^{0}(x) \in L^{1}(-\infty,+\infty)$ and $\xi(t)=t+x^{0}$ satisfies the assumptions of Proposition 5.4. If $d_{n}=2^{-n} \exp (-V-2 n V), n=1,2, \ldots$, then $e^{V x} u^{0}(x)$ $\in L^{1}(-\infty,+\infty)$ and then Proposition 5.5 is applicable.

Example 5.7. Take the same function $a(x)$ as in Example 5.6. Put

$$
u^{0}(x)= \begin{cases}0, & x<1, \\ b_{n} a\left((x-2 n) / d_{n}\right), & 2 n-1 \leqq x \leqq 2 n+1,\end{cases}
$$

with $d_{n}=2^{-n} \exp (-V-2 n V), n=1,2, \ldots$. Here $b_{n} \leqq 1$ are to be determined according to the choice of $v^{0}(x)$. For example, if $v^{0}(x)=2 V /(x+1)$ for $x>0$, then $b_{n}$ can be chosen as $b_{n}=(2 n+1)^{-\alpha}, 0 \leqq \alpha<1$. Then $u(n, n) \rightarrow$ $+\infty$ as $n \rightarrow+\infty$.

Remark 5.8. Note that under the assumptions of Proposition 5.5, we have

$$
\int_{0}^{\infty} u(t, t+\eta) d t<+\infty
$$

for every $\eta$ while $u\left(t_{n}, t_{n}+\eta\right) \rightarrow+\infty$ as $n \rightarrow+\infty, t_{n}=\left(\xi_{n}-\eta\right) / 2, \eta \leqq \eta^{0}$. In fact, (21) follows from (9) and $e^{V x} u^{0}(x) \in L^{1}(-\infty,+\infty)$. On the other hand, under the same assumptions we can estimate $v(t, t+\eta)$ from below. In fact, by (4) we have 


$$
\begin{aligned}
& v(t, t+\eta) \\
& \geqq v^{0}(\eta) \exp \left\{-\frac{1}{2} \int_{\eta}^{\xi} u^{0}(\theta) \exp \left(\frac{1}{2} \int_{\eta}^{\theta} v^{0}(\sigma) d \sigma\right) d \theta\right\} \\
& \geqq v^{0}(\eta) \exp \left\{-\frac{1}{2} \int_{\eta}^{\xi} u^{0}(\theta) e^{(\theta-\eta) v} d \theta\right\},
\end{aligned}
$$

$\xi=\eta+2 t$. Since

$$
\int_{\eta}^{\xi} u^{0}(\theta) e^{\theta V} d \theta \leqq \int_{-\infty}^{\infty} u^{0}(\theta) e^{\theta V} d \theta=C
$$

we have

$$
v(t, t+\eta) \geqq v^{0}(\eta) \exp \left\{-C e^{-\eta V}\right\} .
$$

Here the right hand side is independent of $t$.

\section{References}

[1] Mimura, M., Dissertation, Kyoto University (Fac. of Eng.) 1973.

[2] Mimura, M., Kametaka, Y., and Yamaguti, M., On a certain difference scheme for some semilinear diffusion system, Proc. Japan Acad., 47 (1971), 385-387.

[ 3 ] Yamaguti, M., On Boltzmann model and Volterra model, Sûri-Kaiseki-Kenkyûsho Kôkyûroku, 174, Kyoto University (1973) (in Japanese). 
